\title{
Diversité des mécanismes de résistance aux chimiothérapies anticancéreuses
}

La résistance des cellules tumorales aux agents cytotoxiques représente un obstacle majeur de la chimiothérapie anticancéreuse. Les mécanismes en cause sont multiples, extrinsèques (liés aux relations hôte-tumeur ou hôte-médicaments) ou intrinsèques. Parmi ces derniers, on peut noter des altérations du transport membranaire des produits cytolytiques, leur inactivation métabolique ou la modification de leurs cibles les rendant résistantes, une augmentation des phénomènes de réparation de l'ADN ou de détoxication des intermédiaires réactifs engendrés par les chimiothérapies. Souvent, la résistance des cellules cancéreuses est pléïotrope, due à plusieurs mécanismes indépendants. Elle pourrait être engendrée à la fois par sélection clonale et par des phénomènes adaptatifs directement induits par les anticancéreux.

\section{Dominique Belpomme}

\section{ADRESSE}

D. Belpomme : praticien hospilalier, directeur $d u$ centre de recherche de l'ARTAC (Association pour la recherche thérapeutique anticancéreuse). Unitć de chimiothérapie, hôpital Bichat, 46, rue Henri-Huchard, 75018 Paris, France. e projet téléonomique* d'une cellule eucaryote normale est sa différenciation ; celui d'une cellule cancéreuse, sa division. Telle est, de façon schématique, la différence fondamentale entre cellules normale et cancéreuse. L'acquisition d'une capacité de division illimitée confère un état de malignité à la cellule qui en est la cible. Un tel état rejoint, par certains aspects, le projet téléonomique des cellules procaryotes, capables de se diviser rapidement, mais incapables de se différencier et de communiquer entre elles, même

\footnotetext{
* Projet téléonomique: peut s'entendre par "but
} final " ou "objectif". au sein des colonies qu'elles sont susceptibles de former.

La résistance thérapeutique peut donc être définie comme la persistance d'un projet de division après traitement.

En cancérologie, la résistance est souvent évaluée par rapport aux phénomènes de cytotoxicité : sont considérées être résistantes, les cellules non détruites, ayant conservé leur capacité de prolifération. Compte tenu de la possibilité de perte du pouvoir clonogène de certaines cellules au cours du développement tumoral, cytotoxicité et résistance n'ont une signification thérapeutique que si les cellules souches tumorales sont seules concernées.

La diversité des thérapeutiques anti- 


\section{RÉFÉRENCES}

1. Heppner G. Tumor heterogeneity. Cancer Res 1984 ; 44 : 2259-65.

2. Jones $\mathrm{P}$, Taylor S. Cellular differentiation : cytidine analogs and DNA methylation. Cell 1980 ; 20 : 85-93.

3. Skipper H, Perry S. Kinetics of normal and leukemic leukocyte populations and relevance to chemotherapy. Cancer Res $1970 ; 30: 1883-97$

4. Valeriote F, Van Putten L. Proliferationdependent cytotoxicity of anticancer agents : a review. Cancer Res 1975; 35 : 2619-30.

5. Skipper H, Schabel F, Wilcox W. Experimental evaluation of potential anticancer agents XIV. Further study of certain basic concepts underlying chemotherapy of leukemia. Cancer Chemother Rep 1965 ; 45 : 5-28.

6. Currie G, Currie A. Cancer, the Biology of Malignant Disease. London: Edward Arnold, 1982 : 135.

7. Valeriote F, Edelstein $\mathrm{M}$. The role of cell kinetics in cancer chemotherapy. Semin Oncol $1977 ; 4: 217-26$

8. Chabner B, Collins J. Cancer Chemotherapy: Principles and Practice. Philadelphia: Lipincott JB Company, 1990 : 545.

9. Jain R. Delivery of novel therapeutic agents in tumors : physiological barriers and strategies. JNCI 1989 ; 81 : 570-6.

10. Vanpel $P$, Kallinowski F, Okunieff $P$ Blood flow, oxygen and nutrient supply and metabolic microenvironment of human tumors: a review. Cancer Res $1989 ; 49$ : 6449-65.

11. Brown J. Tumor hypoxia, drug resistance and metastases. JNCI 1990; 82 : 338-9.

12. Hall T. Prediction of responses to therapy and mechanisms of resistance. Semin Oncol $1977 ; 4$ : 193-202.

13. Curt G, Clendennin N, Chabner B Drug resistance in cancer. Cancer Treat Rep cancéreuses et leur possibilité de modifier le phénotype tumoral par des mécanismes autres que purement génétiques [1,2] explique que cette définition par référence aux phénomènes de cytotoxicité soit trop restrictive. Avec le cancer, la résistance concerne, en effet, non seulement les thérapeutiques cytotoxiques classiques, mais aussi celles qui agissent par d'autres mécanismes.

Bien qu'il ne semble pas exister d'argument biologique suffisamment solide pour distinguer fondamentalement la radiorésistance de la chimiorésistance, si ce n'est la plus grande diversité des mécanismes à l'origine de cette dernière, les problèmes concernant la résistance aux agents anticancéreux cytotoxiques classiques font seuls l'objet de cette étude.

Ainsi entend-on ici, sous le terme de " chimiorésistance ", celle concernant les médicaments dont l'effet cytotoxique résulte essentiellement de l'induction directe ou indirecte de lésions au niveau de l'ADN tumoral.

\section{Les différents types de chimiorésistance}

Les études biochimiques in vitro ont conduit à définir sur les plans cellulaire et moléculaire ce qu'il est convenu d'appeler la résistance " intrinsèque ". Appliqué à la situation in vivo, le concept de chimiorésistance a dû être étendu. Interviennent en effet d'autres facteurs qui relèvent de l'hôte et/ou de la tumeur. Ces facteurs expliquent que certaines cellules, résistantes in vivo, ne le soient pas nécessairement ex vivo et qu'inversement certains clones résistants ex vivo ne le soient pas in vivo. Aussi convient-il de distinguer fondamentalement la chimiorésistance intrinsèque, telle que nous l'étudions plus loin, de celle observée cliniquement.

Le Tableau I résume les différents types de chimiorésistance individualisés.

La résistance "cinétique" a été la première correctement étudiée. Les conditions cinétiques de la croissance tumorale [3] et l'éventuelle inadéquation à celle-ci des agents cytotoxiques utilisés, compte tenu de leur(s) différent(s) mécanisme(s) d'action au niveau du cycle cellulaire [4], ont contribué à la définir. Ce type de chimiorésistance résulte du fait que la cytotoxicité des cellules clonogéniques obéit à une cinétique d'ordre 1 (loi du Log cell kill) [5] et donc qu'à chaque administration de chimiothérapie, la totalité des cellules tumorales clonogéniques n'est pas détruite, mais seulement un certain pourcentage. Un tel phénomène est expliqué par le fait que les cellules en G1 sont, de

\footnotetext{
- Différents arguments, dont l'absence de points de restriction en GI, après culture des cellules tumorales clonogéniques en milieu appauvri, conduisent à admet tre l'hypothèse selon laquelle les cellules clonogéniques tumorales, contrairement aux cellules normales, seraient incapables d'entrer en GO, mais resteraient en GI prolongeie [6]. On appelle points de restriction les moments de G1 dans lesquels les cellules normales demeurent. apres synchronisation, induites par les conditions de micro-environnement telles que déplétions en acides aminés. $A$ l'invarse des cellules normales, les cellules tumorales, ne présenteraient pas de tels moments, les conditions de micro-environnement induisant soit leur persistance à n'importe quel moment de G1, soil des lésions irréversibles et leur mort. Les cellules tumorales seraient incapables de passer en GO
}

Tableau 1

LES DIFFÉRENTS TYPES DE CHIMIORÉSISTANCE

\begin{tabular}{|l|l|l|}
\hline \multicolumn{1}{|c|}{ Type } & \multicolumn{1}{|c|}{ Cible } & Méthode d'investigation \\
\hline Cinétique & tumeur (G1) & in vivo/ex vivo \\
\hline Pharmacologique & hôte/tumeur & in vivo/ex vivo \\
\hline Anatomique* & hôte/tumeur & in vivo \\
\hline Biochimique* * $^{*}$ & tumeur & in vitro \\
\hline
\end{tabular}

* Encore appelée : "sectorielle ".

* Encore appelée : "intrinsèque". 
façon générale, moins chimiosensibles que les cellules en phase $\mathrm{S}$ ou $\mathrm{M}^{*}$ (voir note p. 466).

Cet aspect purement cinétique - mis en évidence chez l'animal, à partir de modèles tumoraux "liquides" (leucémies) ou solides, le plus souvent greffables et à temps de doublement rapide - a longtemps résumé l'ensemble des idées que l'on se faisait de la chimiorésistance en cancérologie [7]. Des critiques sont cependant apparues, mettant en doute une telle conception uniciste. D'autres facteurs sont en effet à considérer : pour être cytotoxiques in vivo, les médicaments doivent être administrés à une dose seuil critique, certains d'entre eux nécessitant d'être activés par l'hôte ou les cellules tumorales. L'administration de doses inférieures à ce seuil critique, l'utilisation de voies d'administration inappropriées, l'absence ou l'insuffisance d'activation de ces médicaments ont conduit à définir une autre sorte de chimiorésistance, appelée "pharmacologique " [8].

Par ailleurs, le site anatomique de la tumeur et ses caractéristiques d'organisation interne, notamment architecturaux et vasculaires, conditionnent la perméabilité des cellules tumorales aux médicaments. L'existence chez l'hôte de sanctuaires naturels [9] et, au sein des tumeurs solides, d'un stroma (tissus sains péri- et intratumoraux), plus ou moins perméables à ces agents [10] définissent ce que nous appelons la résistance " anatomique " ou "sectorielle ". Ainsi la mise en évidence d'une "barrière hémato-méningée " pour de nombreux agents cytotoxiques administrés par voie systémique explique-t-elle la relative chimiorésistance des localisations cérébro-méningées des cancers et leur possible réensemencement systémique à partir de ces sites. De même l'altération du microenvironnement tumoral (stroma) par la radiothérapie, explique-t-elle la chimiorésistance observée lorsque la tumeur siège en territoire irradié.

L'individualisation de ces différents types de résistance est certes arbitraire, compte tenu de leur étroite interdépendance, mais elle a pour mérite de clarifier les différents problèmes posés. A titre d'exemple, l'hypoxie intratumorale, bien étudiée $\mathrm{m} / \mathrm{s} n^{\circ} 5$, vol. 7 , mai 91

Tableau II

ALTÉRATION DU TRANSPORT TRANSMEMBRANAIRE DES AGENTS ANTICANCÉREUX

Diminution de flux entrant dépendant d'un transporteur

- Méthotrexate

- Agents alkylants melphalan : système "leucine" mechloréthamine: système "choline"

- Sels de platine

Augmentation de flux sortant actif à dépendance énergétique

- Alcaloïdes de pervenche (vincristine, vinblastine, vindésine)

- Alcaloïdes de la podophylle (étoposide, ténoposide)

- Intercalants : anthracyclines (doxorubicine) anthracénediones (mitoxantrone)

- Autres antibiotiques: actinomycine $\mathrm{D}$ mitomycine $C(?)$

en radiobiologie par la mise en évidence de "l'effet oxygène ", est aussi une cause, probablement non négligeable, de résistance à la chimiothérapie [11]. Or si différents facteurs y contribuent, dont le degré de vascularisation tumorale, les conséquences qui en résultent sur le plan de la résistance sont en réalité multiples, génétiques (induction d'anomalies chromosomiques) et biochimiques, mais aussi cinétiques (mise en G1 des cellules) et pharmacologiques.

\section{Diversité des mécanismes de résistance intrinsèque}

La reproduction in vitro du phénomène de chimiorésistance à l'aide de lignées cellulaires normales ou tumorales a permis d'en aborder l'étude sous un jour nouveau. La résistance intrinsèque est en fait un phénomène complexe, dépendant non seulement de la lignée cellulaire concernée, mais aussi du type d'agent cytotoxique utilisé.

De nombreuses revues générales ont été consacrées à ce sujet $[8,12,13]$. Nous ne pouvons en donner ici qu'un aperçu. Schématiquement, cing grands mécanismes peuvent être individualisés : (1) l'altération du transport transmembranaire des agents anticancéreux; (2) l'altération quantitative et/ou qualitative de leurs protéines cibles; (3) l'altération quantitative et/ou qualitative des enzymes d'activation ou d'inactivation impliquées dans leur métabolisme; (4) l'altération quantitative et/ou qualitative des processus de détoxication cellulaire, dont on peut rapprocher (5) l'activation des systèmes de réparation de l'ADN.

\section{- Altération du transport trans- membranaire}

L'altération du transport transmembranaire des agents cytotoxiques est l'un des mécanismes les mieux étudiés (Tableau II). Une diminution du flux entrant transmembranaire a été mise en évidence pour certains antimétabolites tels le méthotrexate, certains sels de platine et certains alkylants.

Alors que ce transport est essentiellement passif pour les nitroso-urées, en raison de leur lipophilie, celui-ci dépend d'un transporteur pour d'autres alkylants, qui empruntent soit le système "choline" (méthochloréthamine) [14], soit le système "leucine" (melphalan) [15]. A l'inverse, une augmentation du flux sortant transmembranaire, à dépendance énergétique, a été individualisée pour certaines molécules naturelles de grande taille, incluant les intercalants anthracycliniques, certains antibiotiques tels l'actinomycine D, les alcaloïdes de la pervenche et de la podophylle.

J. Biedler et V. Ling ont d'abord 


\section{Enzymes}

Dihydrofolate réductase (DHFR) : méthotrexate

Thymidylate synthétase : 5-fluoro-uracile

Ribonucléotide réductase : hydroxyurée

Topo-isomérase $\|$ : amsacrine, anthracyclines (doxorubicine), anthracènediones (mitoxantrone), ellipticines, alcaloïdes de la podophylle (vépéside, téniposide)

\section{Protéines de structure}

Tubuline : alcaloïdes de pervenche (vincristine, vinblastine, vindésine) Récepteurs membranaires: anthracyclines (doxorubicine)

14. Coldenberg GJ, Vanston CL, Bihler I. Transport of nitrogen mustard on the trans port carrier for choline in L 5178 Y lymphoblasts. Science $1971 ; 172$ : 1148-9.

15. Begleiter A, Lam HYP, Grover J, et al. Evidence for active transport of melphalan by two amino acid carriers in L 5178Y lymphoblasts in vitro. Cancer Res $1979 ; 39$ : 353-9.

16. Biedler J, Richm H. Cellular resistance to actinomycin $\mathrm{D}$ in Chinese hamster cells in vitro : cross-resistance, radioautographic and cytogenetic studics. Cancer Res 1970 ; 30 : 1174-84.

17. Ling $\mathrm{V}$, Thompson LH. Reduced permeability in $\mathrm{CHO}$ cells as a mechanism of resistance to colchicine. J Cell Physiol 1973 ; 83 : 103-16.

18. Dano K. Active outward transport of dannomycin in resistant Ehrlich ascites tumor cells. Biochim Biophys Acta 1973; 323 : 1466-83

19. Inaba $M$, Kobayashi $H$, Sakurai $Y$ Johnson R. Active efflux of daunorubicin and adriamycin in sensitive and resistant sublines of P388 leukemia. Cancer Res 1979 ; 39: 2200-3

20. Riordan J, Ling V. Purification of Pglycoprotein from plasma membrane vesicles of Chinese hamster ovary cell mutants with reduced colchicine permeability. $J$ Biol Chemistry 1979 ; 254 : 12701-5.

21. Deuchars K, Ling V. P-glycoprotein and multidrug resistance in cancer chemotherapy. Semin Oncol 1989; $16: 156-65$

22. Marie JP. Le phénomène de résistance multiple aux anticancéreux : les gènes MDR et la pGp. médecine/sciences $1990 ; 6$ : 443-8.

23. Schimke R, Alt F, Kellems R, Kaufman R, Bertino J. Amplification of hydrofolate reductase genes in methotrexateresistant cultured mouse cells. Cold Spring Harbor Symp Quant Biol 1978 ; 42 : 649-57.

24. Flintoff WF, Essani K. Methotrexateresistant Chinese hamster ovary cells contain a dihydrofolate reductase with an altered affinity for methotrexate. Biochemistry $1980 ; 19: 4321-7$ observé l'existence d'une diminution de l'accumulation intracellulaire de ces médicaments $[16,17]$. Puis K. Dano et M. Inaba ont montré que le défaut d'accumulation résultait plus d'une augmentation du flux sortant actif que d'une diminution du flux entrant $[18,19]$, bien que ce dernier mécanisme puisse coexister. La mise en évidence d'une résistance croisée spécifique à ces différents médicaments et l'absence habituelle de résistance croisée de ceux-ci visà-vis des alkylants, sels de platine et antimétabolites a par ailleurs conduit J. Biedler à individualiser le classique phénotype MDR (multidrug resistance) de résistance multiple aux drogues [16].

La détection puis la purification par V. Ling [20] d'une glycoprotéine particulière, la pGp 170, présente à l'état naturel dans la membrane de certaines cellules normales et en quantité augmentée dans celle des cellules résistantes, ont en outre permis de la caractériser structurellement et d'expliquer son mécanisme d'action [21, 22].

L'homologie de structure entre la pGp 170 et l'hémolysine B des bactéries suggère en effet le rôle de " pompe refoulante" de cette glycoprotéine, bien qu'une autre explication faisant intervenir une augmentation de $\mathrm{pH}$ membranaire ait été récemment proposée par T. Skovsgaard. En fait, comme nous le reverrons, l'individualisation du phénotype MDR ne peut résumer à lui seul, dans sa forme "typique ", l'ensem- ble des phénomènes de résistance pléïotropique observés.

- Altération quantitative et/ou qualitative des protéines cibles L'augmentation et/ou l'altération qualitative de différentes enzymes ou protéines de structure cellulaire, cibles de certains agents anticancéreux, est un deuxième mécanisme de résistance. L'augmentation de la dihydrofolate réductase (DHFR) secondaire à l'administration de méthotrexate est sans doute le modèle le mieux étudié [23]. Il a permis d'établir que l'augmentation intracellulaire stable de cette enzyme, par ailleurs structurellement normale, peut conférer la résistance par un mécanisme d'amplification génique. Cependant, l'altération qualitative de cette enzyme par mutation [24] est aussi possible, la perte d'affinité de l'enzyme pour son substrat (le méthotrexate) expliquant la résistance. En fait, de telles altérations quantitatives et/ou qualitatives ont été observées avec d'autres antimétabolites, dont le 5-fluoro-uracile et l'hydroxyurée [13].

La découverte récente que la cytotoxicité au niveau de l'ADN pouvait dépendre de différentes enzymes, dont les topo-isomérases I (rupture d'un seul brin d'ADN) et II (rupture des deux brins d'ADN) [25] a singulièrement élargi le champ d'étude des résistances et conduit à individualiser un deuxième type de résistance MDR. Dans le cas de la topoisomérase II, certains médicaments, 
dont les anthracyclines, les anthracènediones (mitoxantrone), les alcaloïdes de la podophylle, les ellipticines et les acridines (amsacrine) sont en effet susceptibles de stabiliser le complexe clivable ADN-enzyme, la conséquence en étant une cassure relativement stable de l'ADN et donc dans certains cas, la mort cellulaire. Or, bien que la synthèse de la topoisomérase II soit dépendante du cycle cellulaire, des altérations quantitatives (diminution) et/ou qualitatives (altération catalytique) de cette enzyme nucléaire ont pu être observées en cas de résistance aux médicaments précités [26].

L'altération qualitative de certaines protéines de structure cellulaires, cibles des alcaloïdes de pervenche (tubuline) ou d'intercalants anthracycliniques (récepteurs membranaires lipidiques), constitue enfin une autre éventualité, témoignant de la très grande diversité des phénotypes observés [13] (Tableau III).

- Altération quantitative et/ou qualitative d'enzymes impliquées dans le métabolisme des agents anticancéreux

L'altération du métabolisme des agents cytotoxiques par diminution d'activité des enzymes d'activation ou, ou contraire, par augmentation d'activité des enzymes "d'inactivation " est un troisième mécanisme de chimiorésistance possible. La diminution de la polyglutamatation du méthotrexate (les polyglutamates sont une forme de stockage intracellulaire du médicament) est un exemple classique [27]. En outre, des anomalies quantitatives ou même qualitatives de plusieurs autres enzymes intervenant dans le métabolisme des antimétabolites (cytosine-arabinoside, 6-thiopurines, etc.), de certains antibiotiques (bléomycine) ou même des anthracyclines ont été mises en évidence (Tableau IV). Ainsi la perte de réduction des quinones anthracycliniques en semiquinones diminue la production de radicaux libres et explique la diminution d'efficacité de ces médicaments en raison de leur capacité moindre à altérer l'ADN cellulaire [13].

- Altération quantitative des protéines de détoxication cellulaire L'augmentation de synthèse des pro$m / s n^{\circ} 5$, vol. 7 , mai 91
Tableau IV

ALTÉRATION DES ENZYMES IMPLIQUÉES DANS LE MÉTABOLISME DES AGENTS ANTICANCÉREUX

\section{Diminution d'activation métabolique}

\section{Activité enzymatique diminuée}

Désoxycytidine kinase : cytosine-arabinoside

Uridine cytidine kinase : 5-azacytidine

Orotic acid phosphoribosyl transferase (OAPT) : 5-fluoro-uracile

Uridine phosphorylase : 5-fluoro-uracile

Hypoxanthine guanine phosphoribosyl transférase (HGPRT) :

\section{6-mercaptopurine}

6-thioguanine

Folypolyglutamate synthétase : méthotrexate

P450 réductase, flavine réductase : doxorubicine

\section{Augmentation d'inactivation métabolique}

\section{Activité enzymatique augmentée}

Cytidine désaminase, dCMP désaminase : cytosine arabinoside

Phosphatase alkaline:

6-mercaptopurine

6-thioguanine

Hydrolase : bléomycine

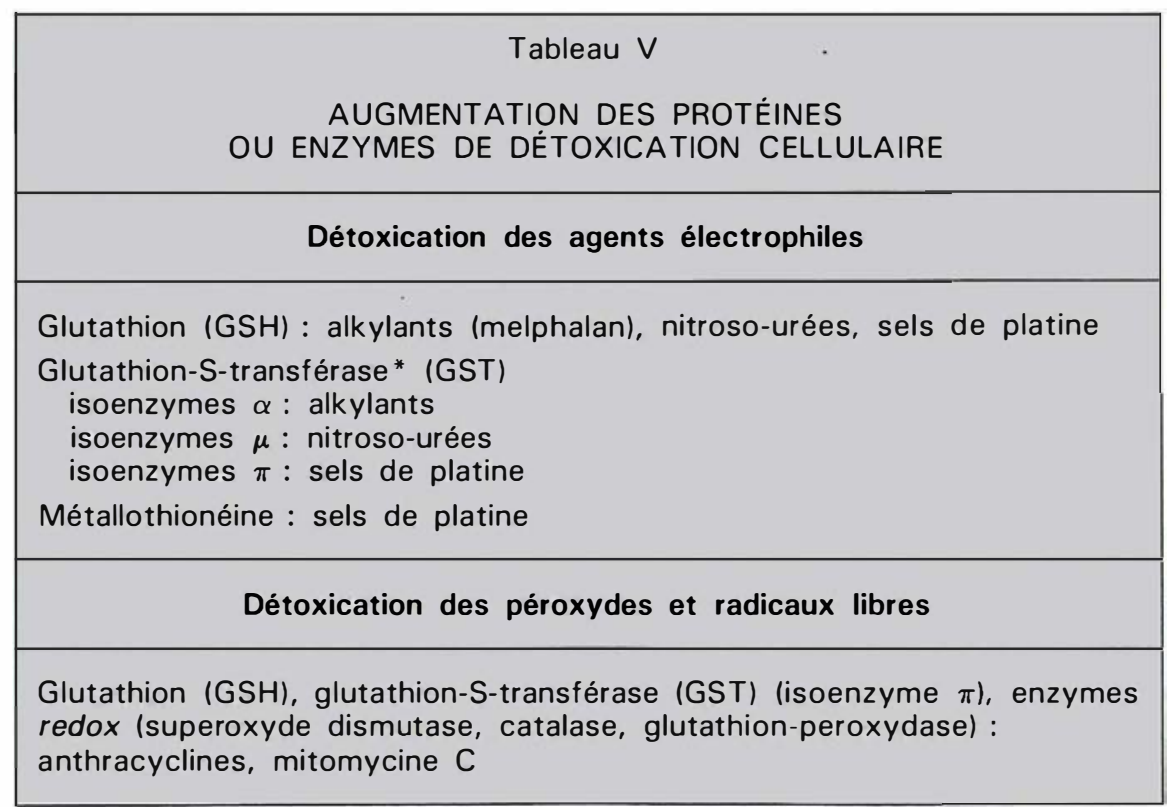

* Les isoenzymes $\alpha, \mu$ et $\pi$ sont respectivement situées sur les chromosomes 6p12, $1 p 31$ et $11 q 13$. 


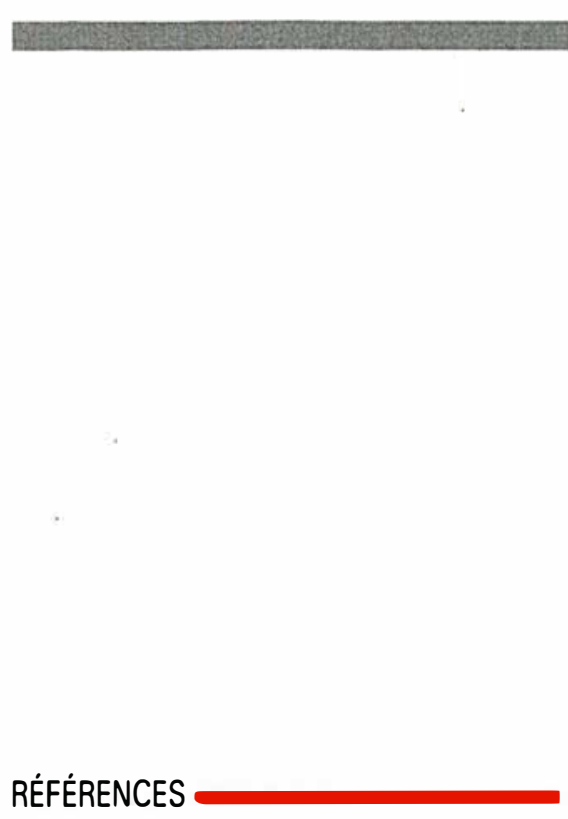

25. Holm C, Covey J, Kerrigan D, Pommier Y. Differential requirement of DNA replication for the cytotoxicity of DNA topoisomerase I and II inhibitors in Chinese hamster DC3F cells. Cancer Res $1989 ; 49$ : 6365-8.

26. Beck W. Unknotting the complexities of multidrug resistance : the involvement of DNA topoisomerase in drug action and resistance. JNCI $1989 ; 81$ : 1683-5.

27. Cowan KH, Jolivet J. A novel mechanism of resistance to methotrexate in human breast cancer cells : lack of methotrexate polyglutamate formation. Clin Res 1983 ; $31: 508 \mathrm{~A}$.

28. Cohen J, Lyon R. Multinuclear NMR study of the metabolism of drug sensitive and drug-resistant human breast cancer cells. Ann NY Acad Sci 1987 ; 508 : 216-28.

29. Goldie J, Coldman A. A mathematic model for relating the drug sensitivity of tumors to their spontaneous mutation rate. Cancer Treat Rep 1979; 63 : 1727-33.

30. Belpomme D, Heritier F, Ressayre C. Goldie-Coldman hypothesis revisited : an induction-selection theory of chemoresistance. Proc Am Soc Clin Oncol $1989 ; 8$ : 81.

31. Goldie J, Coldman A. The genetic origin of drug resistance in neoplasms : implications for systemic chemotherapy. Cancer Res 1984; 44 : 3643-53.

32. Herweijer $\mathrm{H}$, Sonneveld $\mathrm{P}$, Baas F, Nooter K. Expression of $\mathrm{mdr} 1$ and $\mathrm{mdr} 3$. Multidrug resistance genes in human acute and chronic leukemias and association with stimulation of drug accumulation by cyclosporine. JNCI 1990 ; 82 : 1133-40.

33. Etiemble J, Bernard JF, Picat CH, Belpomme D, Boivin P. Red blood cell enzyme abnormalities in patients treated with che-

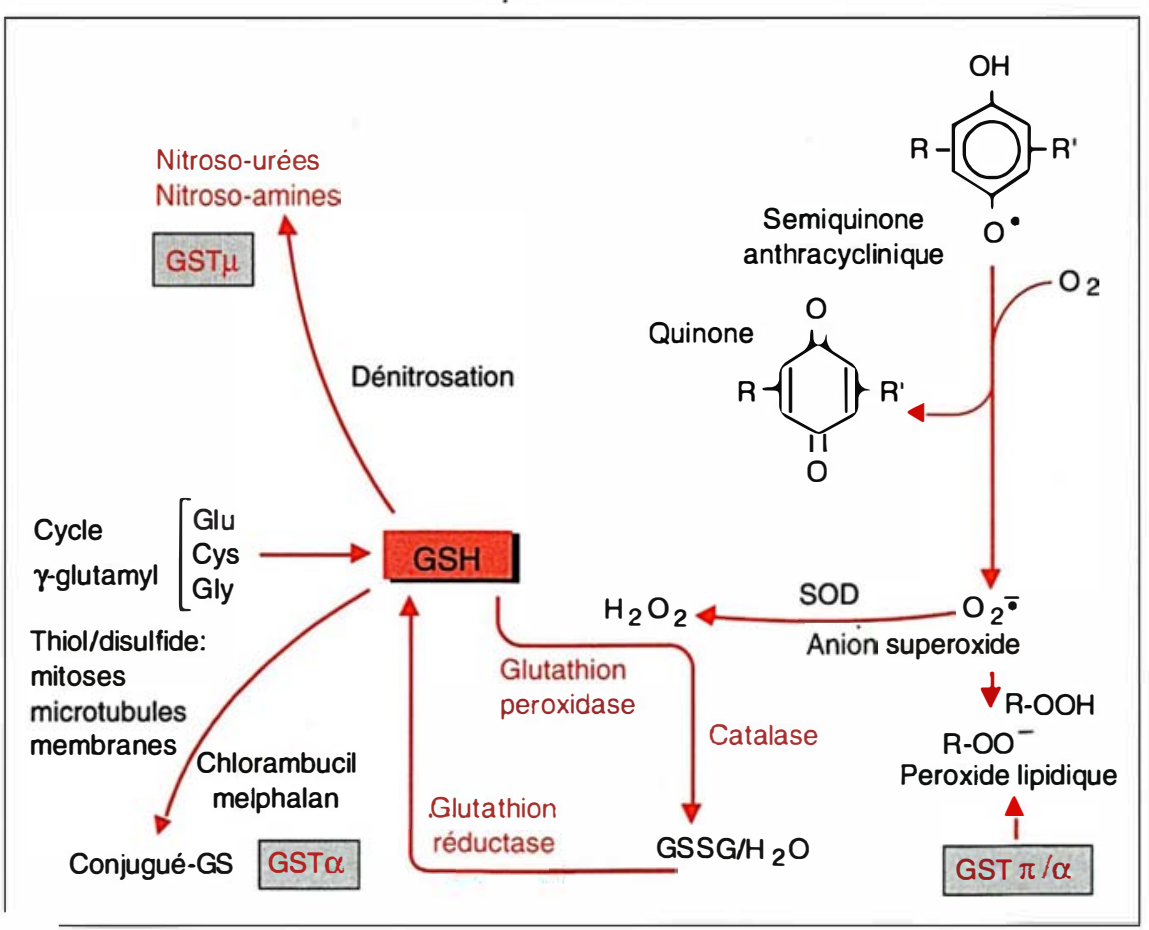

Figure 1. Schéma simplifié de l'intervention du glutathion (GSH) et de certaines enzymes dans les processus de détoxication cellulaire. Glutathion-S-transférases (GST) $\alpha$ et $\mu$, superoxyde dismutase (SOD), glutathion peroxydase, glutathion réductase, catalase, etc.

téines de détoxication cellulaire, tels le glutathion (GSH) ou la métallothionéine, ou d'enzymes de détoxication, telles les glutathion-Stransférases (GST) et les enzymes redox, est un quatrième mécanisme possible de chimiorésistance (Tableau V, p. 469). Celui-ci concerne essentiellement les alkylants, nitrosourées et sels de platine ainsi que certains intercalants [13]. Une augmentation du glutathion intracellulaire est en effet observée en cas de résistance au melphalan, aux nitroso-urées ou aux anthracyclines, et une augmentation de la métallothionéine en cas de résistance aux sels de platine. C'est aussi dans le cadre de ces processus de détoxication que l'on aurait $\mathrm{pu}$ inclure l'augmentation de synthèse de la pGp 170, puisque cette glycoprotéine exerce un rôle de détoxication cellulaire, en raison, comme nous l'avons vu, de sa fonction de pompe membranaire permettant l'extrusion active des molécules cytotoxiques.
- Activation des systèmes de réparases

L'activation des systèmes de réparases au niveau de l'ADN cellulaire constitue sans doute le mécanisme de chimiorésistance le plus fréquent et aussi, probablement, le plus important de ceux que nous avons envisagés. Il demeure malheureusement le moins bien étudié, notamment chez les eucaryotes, et plus particulièrement chez l'homme où, cependant, différents gènes (ou groupes de gènes) de réparation ont été individualisés: les gènes XRCC1 et XRCC2 $(X$ rays repair cross complementing 1 et 2) présents sur les chromosomes 7 et 19, les gènes ERCC1 et ERCC2 (gènes excision repair cross complementing), présents sur le chromosome 19 et les gènes NER (nucleotide excision repair) présents sur les chromosomes 2, 13, 16 et 19 . De nombreux arguments, directs ou indirects, plaident en faveur de l'importance de l'activation des systèmes de réparation dans la genèse, non $m / s n^{\circ} 5$, vol. 7 , mai 91 
seulement des radiorésistances, mais aussi des résistances aux agents cytotoxiques, en particulier aux alkylants, nitrosourées et intercalants. Récemment, le rôle dans la résistance aux chloro-éthyl-nitroso-urées, de différentes enzymes de réparation, dont celui de la guanine-O-6-alkyl transférase, a été souligné, mais de nombreuses recherches restent encore à faire dans ce domaine.

\section{Signification biologique et clinique de la chimiorésistance}

L'extrême diversité des mécanismes précédents suggère que la résistance intrinsèque est un phénomène complexe, mettant en jeu de façon constitutive ou adaptative un nombre relativement élevé de systèmes moléculaires présents naturellement dans les cellules. Si l'on fait abstraction des phénomènes de perméabilité et de détoxication membranaire, trois groupes d'agents cytotoxiques peuvent être distingués

- Les alkylants, intercalants et médicaments apparentés, dont l'action au niveau de l'ADN est directe et vraisemblablement multifocale, matérialisée le plus souvent par l'existence de "lésions primaires encombrantes" (adduits) puis par celle de cassures mono- ou double brin de l'ADN (lésions secondaires). Les mécanismes de chimiorésistance sont essentiellement ici, comme pour la radiorésistance, l'activation des systèmes de réparation de l'ADN et, de détoxication cellulaire (glutathion, métallothionéine) et, de façon particulière aux intercalants et aux alcaloïdes de la podophylle, la disparition ou l'inactivation de la topoisomérase II.

- Les antimitotiques (alcaloïdes de pervenche), dont le point d'impact concerne essentiellement le fuseau cellulaire, la conséquence en étant un blocage de la division cellulaire ou son altération au moment de la ségrégation chromosomique. Le mécanisme de la résistance semble être ici lié à l'altération d'une seule et même protéine cible : la tubuline.

- Les antimétabolites, dont l'action au niveau de l'ADN est soit directe par incorporation, mais alors le plus $\mathrm{m} / \mathrm{s} n^{\circ}$ 5, vol. 7, mai 91 souvent facilement réparée (5-fluorouracile, cytosine-arabinosine, etc.), soit plus souvent indirecte, par inhibition de la réplication de l'ADN. La chimiorésistance est dans ce cas essentiellement de type métabolique (inhibition ou activation enzymatique) et/ou concerne les enzymes cibles impliquées dans la réplication de l'ADN (DHFR, thymidylate synthétase, etc.).

La classification que nous proposons ici n'est en fait qu'arbitraire. A un médicament donné peuvent en effet correspondre plusieurs mécanismes de chimiorésistance. Ainsi deux mécanismes au moins sont susceptibles d'expliquer la résistance aux alcaloïdes de pervenche : l'augmentation du flux sortant actif transmembranaire et l'altération qualitative de la tubuline. Trois mécanismes au moins peuvent rendre compte de la résistance aux alkylants : l'altération du transport actif transmembranaire, l'activation des systèmes de réparases et celle des systèmes de détoxication. Quatre mécanismes peuvent être responsables de la résistance au méthotrexate: l'altération du transport actif transmembranaire, l'altération quantitative et/ou quantitative de la DHFR, l'altération de la polyglutamatation. Cinq mécanismes, enfin, peuvent être en cause dans la résistance à la doxorubicine: l'altération de récepteurs membranaires, l'augmentation du flux sortant actif transmembranaire, la diminution de la production des radicaux libres, l'augmentation du glutathion intracellulaire et l'inhibition de la topo-isomérase II.

L'extrême diversité de ces altérations pose le problème de leur coexistence ou non dans une même cellule et de la nature des anomalies génétiques (ou extragénétiques) en cause. La rareté des études biochimiques exhaustives des variants obtenus in vitro rend difficile l'interprétation des résultats précédents. Néanmoins, certaines études indiquent que la coexistence de plusieurs phénotypes de résistance au sein d'une même cellule est non seulement possible, mais probablement fréquente et souvent multiple. C'est ce que suggère, par exemple, l'étude de la lignée MCF-7, rendue résistante à la doxorubicine, où le classique phénotype MDR coexiste non seulement avec une aug- mentation de la glutathion-Stransférase et de la glutathion peroxydase, mais aussi avec une diminution de la catalase et de la DTdiaphorase, des anomalies de transport transmembranaire et une augmentation de la glycolyse aérobie [28]. Une telle multiplicité et diversité des altérations enzymatiques dans une même cellule résistante pose à l'évidence le problème de l'existence de plusieurs anomalies génétiques. La théorie génétique a été la première proposée pour rendre compte du phénomène de résistance. Bien que non spécifique, l'amplification génique en est l'altération la plus fréquemment observée ex vivo. Son rôle dans la résistance au méthotrexate a été reconnu dès 1978 par R. Schimke [23]: l'augmentation intracellulaire de DHFR est la conséquence d'une amplification proportionnelle de son gène de structure, et des variants cellulaires possédant jusqu'à 1000 copies ont pu être isolés ex vivo après augmentation progressive de méthotrexate.

$\mathrm{Si}$ le mécanisme séquentiel de l'amplification peut expliquer que plusieurs gènes soient simultanément répliqués, il n'est cependant pas certain que tous les gènes de résistance le soient sur le même segment d'ADN amplifié. Les possibilités de délétions ou de mutations ponctuelles, et surtout celles d'activation ou d'inactivation de certains gènes, constituent d'autres mécanismes possibles de résistance.

Si les expériences d'hybridation cellulaire, et surtout celles de transfection cellulaire utilisant des séquences d'ADN clonées ou même des gènes de résistance purifiés, ont apporté des arguments majeurs en faveur de la théorie génétique de la résistance, sa valeur in vivo nécessite d'être confirmée.

Goldie et Coldman ont proposé, en 1979, un modèle fondé sur l'hypothèse que la résistance est un phénomène génétique, résultant de la survenue spontanée et aléatoire de mutations au cours de la progression tumorale [29]. Dans ce modèle, la résistance est le résultat de la sélection stochastique de mutants naturellement résistants, les agents cytotoxiques n'exerçant qu'une pression neutre de sélection. 
L'observation majeure qui découle de l'expérience clinique usuelle est l'extrême fréquence et la très grande rapidité d'apparition des résistances pléiotropiques après chimiothérapie. Ces résistances ne résulteraient pas tant de l'émergence de plusieurs clones, différents par leur phénotype de résistance, que plutôt de celle d'un ou de plusieurs clones ayant acquis un phénotype de résistance pléiotropique. Cette très fréquente situation d'échec, même pour des tumeurs de très faible volume et après utilisation précoce de multiples agents cytotoxiques, différents pourtant par leur mécanisme d'action, nous a conduit à remettre en cause la validité du modèle précédent [30]. La probabilité de renouvellement des cellules clonogéniques au sein des tumeurs peut certes donner, selon GoldieColdman, une explication de la fréquence, différente d'un type de tumeur à l'autre, de l'émergence spontanée du phénomène de pléiotropie [31], mais cette explication n'est que théorique, et l'hypothèse stochastique qu'elle sous-tend ne rend qu'imparfaitement compte des données cliniques et biologiques précédentes. En effet, selon Goldie et Coldman, seul un nombre très limité d'événements génétiques (de 1 à 2) est susceptible d'expliquer la pléïotropie. Or, nous l'avons vu, le classique phénotype MDR ne peut rendre compte à lui seul de la très grande fréquence de la pléiotropie. En témoigne la description de nombreux phénotypes, dans lesquels l'augmentation de la pGp 170 est associée à d'autres glycoprotéines de membrane présentes en quantité anormale et/ou à des anomalies enzymatiques. Ainsi, l'amplification du gène $m d r 1$ ou son activation est souvent associée à celle d'autres gènes situés à proximité, tel le gène $m d r 3$ ("famille des gènes $m d r ")$ [32] ou à distance. Si l'importance de ces gènes a pu être contestée expérimentalement, il ne semble pas en être de même in vivo, chez l'homme où, par exemple, la fonction de la glycoprotéine codée par le gène $m d r 3$, semble être aussi, comme pour la pGp 170, celle d'une pompe refoulante [32].

La possibilité de moduler $e x$ vivo le phénotype de résistance selon la procédure de " sélection" utilisée (varia- tion de la dose et des délais de mise en contact des médicaments avec les cellules, utilisation ou non de paliers de dose, etc.) suggère en outre que des processus autres que celui d'une simple sélection sont en jeu, et cela d'autant plus que, comme pour les gènes de différenciation, un certain nombre des gènes de résistance, $y$ compris le gène $m d r 1$, sont inductibles. C'est ce que suggère en effet l'étude des processus de détoxication cellulaire et de réparation.

En réalité, les possibilités de modulations épigénétiques (ou extragénétiques) du phénotype des cellules eucaryotes, par les agents anticancéreux [2], expliquent que le phénomène de résistance puisse recouvrir en cancérologie un concept biologique beaucoup plus étendu que celui utilisé en bactériologie [1]. Ainsi avons-nous été conduit à individualiser, à côté du phénomène de résistance intrinsèque mutationnelle, un type épigénétique ou extragénétique de résistance, notamment en raison de son caractère inductible.

Le modèle de Goldie-Coldman suppose en outre une croissance autonome des clones tumoraux dans l'organisme. Or tel n'est pas le cas, en ce qui concerne les tumeurs solides, en raison de l'existence d'un stroma, et parce que le cancer ne peut plus être considéré comme la prolifération indépendante de ces clones, mais bien comme un ensemble d'écosystèmes cellulaires interréactifs, dans le cadre d'une théorie socioécologique beaucoup plus complexe. Or, c'est dans un tel cadre que pourraient s'intégrer certains aspects de la chimiorésistance [1].

Dans un travail précédent, nous avions montré que les agents cytotoxiques sont capables d'induire de multiples altérations enzymatiques et que certaines d'entres elles sont relativement stables [33]. Les observations cliniques précédentes, l'éventualité de phénomènes de résistance de type "adaptif" et la possibilité d'anomalies enzymatiques assez stéréotypées, persistant après chimiothérapie, nous ont conduit à réfuter l'hypothèse des mutations spontanées et aléatoires, comme seul mécanisme possible d'acquisition de la chimiorésistance et à proposer celle d'un deuxième mécanisme possible, celui d'une induction [30] par les agents cytolytiques, et non seulement d'une sélection

\section{Summary}

Diversity of the resistance mechanisms to anticancer cytotoxic drugs

The resistance of tumoral cells to cytotoxic agents is a major hindrance to anticancer chemotherapy. After defining resistance mechanisms, we will review its different types and mechanisms before discussing its biological and therapeutical significance. The extreme polymorphism of the individualized mechanisms is in contrast to the very high frequency of clinically observed phenomenon of pleiotropism. Aside from the mutational theory proposed by V. Ling from which J. Goldie and A. Coldman designed their model, other mechanisms, genetic or not, are possible. The clinically observed resistance may in fact be the result of a complex process, involving not only clonal selection but also, as we proposed, induction phenomena.

\section{TIRÉS A PART}

D. Belpomme. 